\title{
PSICOLOGÍA
}

\section{Eficacia de la estimulación magnética transcraneal como tratamiento para Síntomas Negativos de la Esquizofrenia}

\author{
Daniela Michelle Arce Escobar¹, Luis Federico Páez Mendoza1, \\ Reinhardt Fischer Amarilla ${ }^{1}$
}

\section{Resumen}

Introducción: El tratamiento de la esquizofrenia ha evolucionado a lo largo de la segunda mitad del siglo pasado, principalmente gracias al desarrollo de distintos fármacos. Si bien es cierto que la aparición de estos contribuyó al tratamiento de los síntomas, la mayoría de los pacientes esquizofrénicos continúan teniendo síntomas residuales.

Entre los síntomas complejos de la esquizofrenia, los síntomas negativos, como la amotivación y el aplanamiento afectivo, siguen siendo algunos de los desafíos más molestos para un tratamiento efectivo y una mejora en el resultado. La estimulación magnética transcraneal repetitiva (rTMS) es una técnica de estimulación cerebral neuromoduladora no invasiva que utiliza la aplicación de pulsos magnéticos a través del cuero cabelludo que conduce a un cambio de excitabilidad en las áreas corticales estimuladas. La rTMS aplicada a la corteza prefrontal dorsolateral (DLPFC) puede modular las vías dopaminérgicas extraestriatales y mesostriatales que pueden contribuir a los síntomas negativos, lo que sugiere que el rTMS puede ser una opción terapéutica prometedora para los síntomas negativos de la esquizofrenia.

Objetivo: Determinar la eficacia de la estimulación magnética transcraneal repetitiva para el tratamiento de síntomas negativos de la esquizofrenia.

Material y Método: Revisión sistemática efectuada en la base de datos SCOPUS. Se trabajó deductivamente utilizando las palabras clave: transcranial magnetic stimulation, schizophrenia, negative symptoms, solicitando que la combinación se encuentre presente en el título, resumen o palabras clave del artículo sin incluir un límite temporal. Inicialmente se obtuvieron 116 artículos de los cuales 78 pertenecían a estudios empíricos publicados entre los años 1999 y 2019. Posteriormente se analizaron los 78 resúmenes de los artículos empíricos aplicándoles los siguientes criterios de inclusión: (1) las muestras cuentan con

1. Universidad Nacional de Itapúa, Facultad de Humanidades, Ciencias Sociales y Cultura Guaraní, Paraguay.

E-mail: michellearcetkd@gmail.com

DOI: $10.26885 /$ rcei.foro.2019.249

Trabajo publicado en acceso abierto bajo Licencia Creative Commons. 


\section{Arce Escobar, D. M. Eficacia de la estimulación magnética transcraneal}

un grupo experimental y grupo control, (2) las muestras están conformadas por pacientes diagnosticados con esquizofrenia, (3) la intervención utiliza la estimulación magnética transcraneal, (4) el texto completo del artículo debe estar disponible en español o inglés. Finalmente, 12 artículos fueron seleccionados en base a los criterios de inclusión para su análisis de texto completo.

Resultados: Los resultados obtenidos muestran una mejora significativa de grupos experimentales en comparación a grupos control en 7 de los 12 estudios. En cuanto a efectos adversos, no se reportaron daños de consideración a excepción de algunos efectos secundarios como fatiga o dolor de cabeza. Es notorio que en los estudios en los que no se lograron reducción de los síntomas negativos (5 estudios), las cantidades de sesiones y duración fueron menores en relación a los estudios con resultados favorables. Lo que demostraría mayor efectividad en tratamientos repetitivos y persistentes.

Conclusiones: En base a los resultados obtenidos, se puede hablar de la rTMS como una técnica que de ser aplicada de manera adecuada muestra mucha efectividad a la hora de tratar síntomas tan complejos como son los síntomas negativos de la esquizofrenia. Si bien resulta necesario de mayores investigaciones para recomendar su aplicación, esta revisión expone a los profesionales un recurso con gran potencial para el futuro de la rehabilitación de la esquizofrenia.

Palabras clave: estimulación magnética transcraneal, esquizofrenia, síntomas negativos.

\section{Referencias}

Buchanan, R. W. (2006). Persistent negative symptoms in schizophrenia: an overview. Schizophrenia bulletin, 33(4), 1013-1022.

Cho, S. S., \& Strafella, A. P. (2009). rTMS of the left dorsolateral prefrontal cortex modulates dopamine release in the ipsilateral anterior cingulate cortex and orbitofrontal cortex. PloS one, 4(8), e6725.

Muñoz, F., \& Ruiz, S. (2007). Terapia cognitivo-conductual en la esquizofrenia. Revista Colombiana de Psiquiatría, 98-110.

Tajima, K., Fernández, H., López, J., Carrasco, J., \& Díaz, M. (2009). Tratamientos para la esquizofrenia. Revisión crítica sobre la farmacología y mecanismos de acción de los antipsicóticos. Actas Esp Psiquiatr, 330-342.

Ziemann, U., Paulus, W., Nitsche, M. A., Pascual-Leone, A., Byblow, W. D., Berardelli, A., \& Rothwell, J. C. (2008). Consensus: motor cortex plasticity protocols. Brain stimulation, 1(3), 164-182. 